\title{
КОНКРЕМЕНТЫ МОЧЕВОЙ КИСЛОТЫ У ДЕТЕЙ: ЭПИДЕМИОЛОГИЯ, ДИАГНОСТИКА, ЛЕЧЕНИЕ И ПРОФИЛАКТИКА (ОБЗОР ЛИТЕРАТУРЫ)
}

\section{URIC ACID URINARY STONES IN CHILDREN: EPIDEMIOLOGY, DIAGNOSTICS, TREATMENT AND PREVENTION (LITERATURE REVIEW) \\ E. Bokova O. Vasyukova}

Summary. Urolithiasis is a common disease both in adults and children. This article discusses the epidemiology, diagnosis, treatment and prevention of uric acid stones formation. Radionegative urates are the most complicated in diagnostics. For this reason it is important to to develop an algorythm for urolithiasis diagnostics with both pediatric urologists and radiologists being involved. Further scientific research is needed in order to estimate the effectiveness of different therapy in patients with uric acid urolithiasis.

Keywords: uric acid stones; calculi; alkalization; urolithiasis; diagnosis; chemolisis.
Бокова Елизавета Олеговна

ФГАОУ ВО Первый Московский государственный медицинский университет имени И. М. Сеченова Минздрава России (Сеченовский Университет) eobokova@gmail.com

Васюкова Олеся Александровна

М.н.С., ФГБНУ «Научно-исследовательский институт морфологии человека» o.vas.93@gmail.com

Аннотация. Уролитиаз является распространенным заболеванием, в том числе среди детского населения. В данной статье рассматриваются вопросы эпидемиологии, диагностики, лечения и профилактики возникновения уратов в детском возрасте. Особую трудность составляет диагностика именно камней мочевой кислоты ввиду их рентгенонегативности. Особого внимания заслуживает разработка алгоритма действий хирурга в зависимости от полученных данных при сотрудничестве с сотрудниками отделений лучевой диагностики. Необходим дальнейший научный поиск также для определения эффективности методов лечения уратов у детей.

Ключевые слова: мочекаменная болезнь; мочевая кислота; ураты; эпидемиология; диагностика; хемолиз.

Наиболее часто уратные кристаллы встречаются у новорожденных, так как мочевая кислота связана с распадом нуклеиновых кислот, который находится на максимальном уровне сразу после рождения и остается на высоком уровне до 2 лет [29]. Примерно в 58,8\% случаев локализацией конкрементов у детей является чашечно-лоханочная система [24], и при патологоанатомическом осмотре обнаруживаются уратные «инфаркты» в мозговом веществе почек [23, с. 293].

Состав мочекислых камней может варьироваться: они могут состоять исключительно из мочевой кислоты или наряду с моногидратом оксалата кальция входить в состав смешанных камней. В первом случае различают безводные, моногидратные (ураты аммония и натрия) и дигидратные камни мочевой кислоты $[22,26]$.

Факторы риска возникновения конкрементов МК можно условно разделить на три основные группы: низкий диурез, высокая концентрация МК в моче и низкий $\mathrm{pH}$ мочи $[3,6,9,10,16,22]$. Причиной низкого объема мочи могут быть климатические особенности, диабет II типа, а также хроническая диарея, приводящая к поте- 


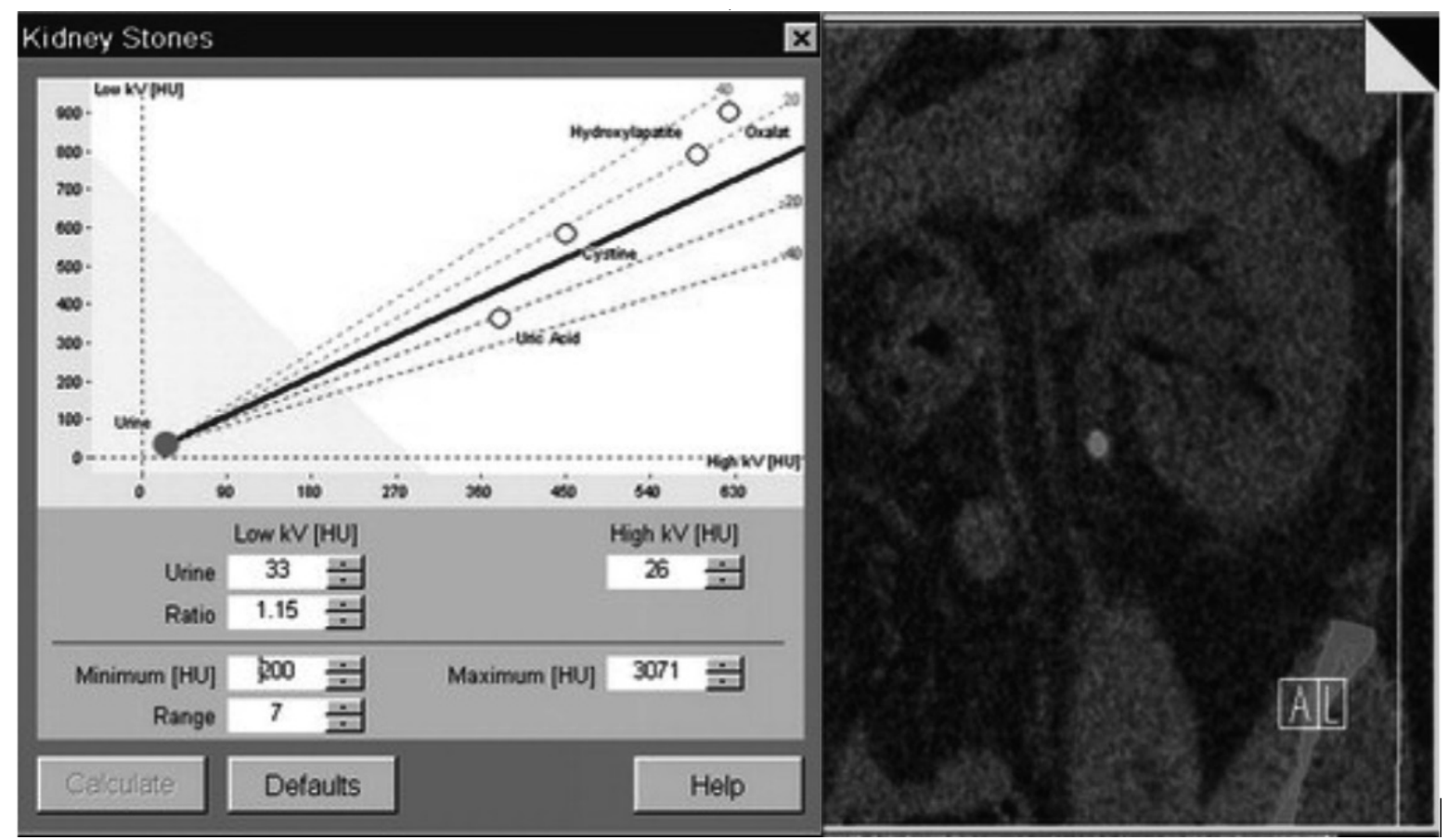

Рис. 1.Использование двухэнергетической КТ для диагностики конкрементов мочевой кислоты, Thomas C. et al. Urinary calculi composed of uric acid, cystine, and mineral salts: differentiation with dual-energy CT at a radiation dose comparable to that of intravenous pyelography //Radiology.— 2010.— T. 257.— № . 2.— C. 402-409. [21].

ре бикарбонатов [3, 22]. Одним из факторов риска возникновения конкрементов МК является жаркий сухой климат со средней летней температурой $45^{\circ} \mathrm{C}$ [3], что объясняет больший процент заболеваемости в южных странах.

Повышение концентрации МК в моче может быть обусловлено как врожденными, так и приобретенными факторами. К первой группе относят такие заболевания как конгенитальные нарушения пуринового обмена и гликогенозы, ко второй - повышенное потребление пуринов с пищей или ускоренный пуриновый обмен на фоне химиотерапии или приема урикозурических медикаментов. Диета с повышенным содержанием мяса, ожирение и диабет II типа, в том числе в составе метаболического синдрома, приводят к снижению рН мочи [6, $9,22]$. Была доказана прямая зависимость между заболеваемостью уролитиазом и индексом массы тела [3, 25].

Факторами риска возникновения уратов у детей является наследственная предрасположенность к нарушению обмена веществ (первичная подагра у детей старшего возраста, синдром Леш-Найана, гликогеноз I типа), а также неверное введение прикорма на первом году жизни и наличие синдрома мальабсорбции $[23,27,29]$. Кроме того, уратный уролитиаз у детей ассоциирован с мутациями белков-транспортеров SLC22A12, SLC2A9 и другими врожденными заболеваниями [29].
Диагностика уролитиаза основана на анамнестических данных, результатах лабораторных и инструментальных методов исследования [14]. Критериями гиперурикозурии является экскреция мочевой кислоты более 4 ммоль/сут у взрослых и более 0,12 ммоль/кг/сут у детей [18]. Камни МК яляются ренгенонегативными конкрементами, однако они могут быть визуализированы при компьютерной томографии без контрастирования. В целях дифференциальной диагностики камней МК от кальциевых конкрементов у взрослых применяется двухэнергетическая КТ (рис. 1) [18, 21], которая однако является довольно дорогостоящим исследованием для применения в рутинной клинической практике. Кроме того, в детском возрасте нежелательно избыточное применение методов лучевой диагностики: предпочтительно выполнение ультразвукового исследования, и лишь при сомнении в диагнозе - КТ без контрастирования или МРТ. В ряде случаев проводится также урография с внутривенным контрастированием [8].

Распространенными инструментальными методами лечения конкрементов МК являются экстракорпоральная ударно-волновая литотрипсия, ретроградная интраренальная хирургия и чрескожная нефролитотрипсия $[16,28]$. При этом, было показано, что волновая литотрипсия является методом выбора у детей до 2-х лет [1]. Yadav P. et al. выработали алгоритм выбора лечебной тактики у детей в зависимости от локализации и размера 


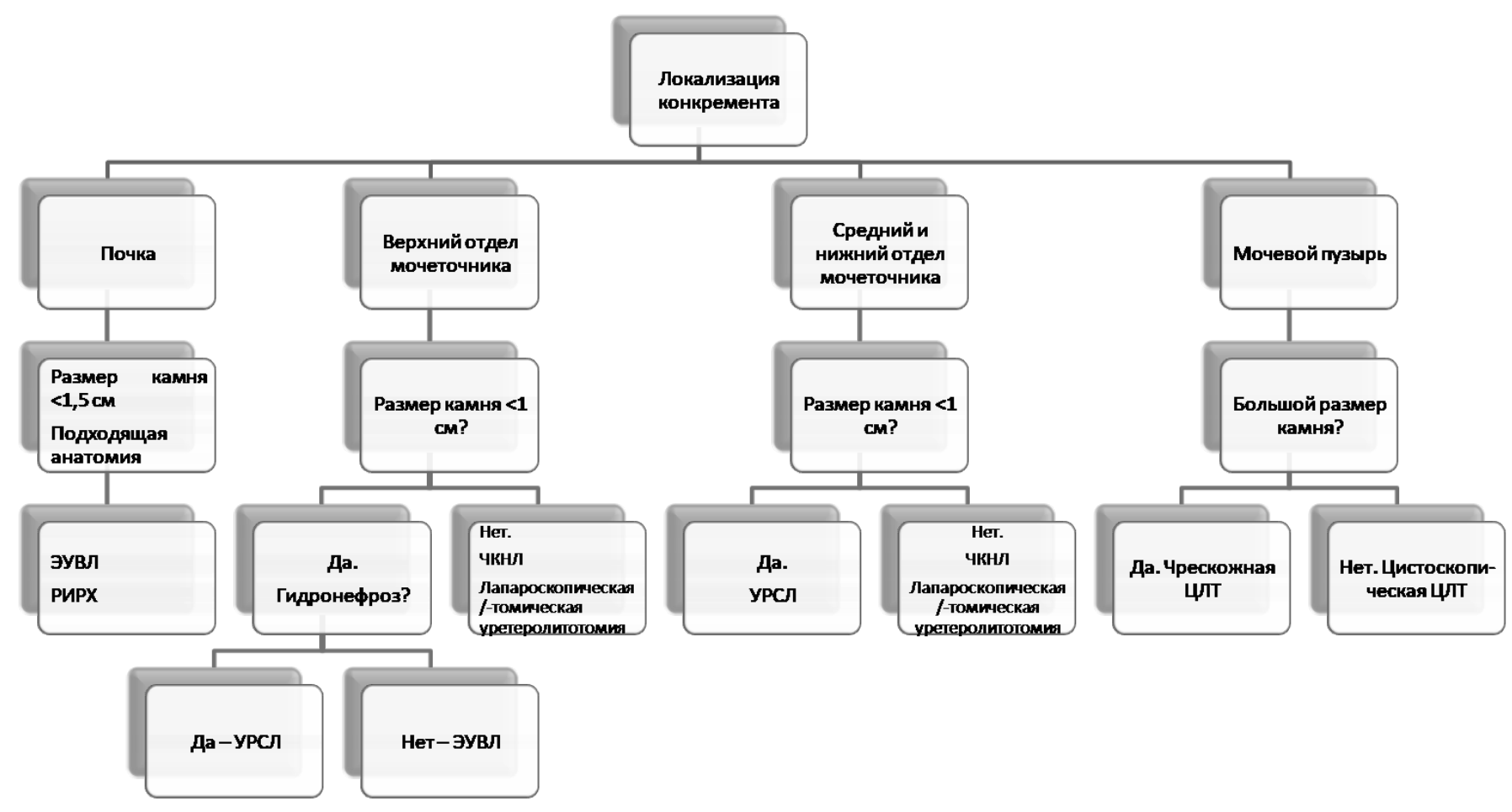

Рис. 2. Выбор метода лечения мочекаменной болезни у детей. ЭУВЛ: экстракорпоральная ударноволновая литотрипсия; РИРХ: ретроградная интраренальная хирургия; ЧКНЛ: чрескожная нефролитотомия; УРСЛ: уретероскопическая литотрипсия; ЦЛт: цистолитотрипсия. Yadav P. et al. Technique, complications, and outcomes of pediatric urolithiasis management at a tertiary care hospital: evolving paradigms over the last 15 years //Journal of pediatric urology.— 2019.— T. 15.— № .6.— C. 665 . e1-665. e7 [24].

камней, используемый в клинической практике (рис. 2) [24].

Альтернативным консервативным способом, применяющимся для лечения данного вида уролитиаза, является растворение уратов (хемолиз). Наряду с пероральным возможно также проведение перкутанного хемолиза [18].

Растворение уратов возможно при рН мочи от 6,5 до 7,0 и выше $[7,18]$. Не рекомендуется повышать $\mathrm{pH}$ мочи выше 7,0, так как это повышает риск возникновения кальциевых конкрементов [17]. Степень растворимости меняется от 7 мг/дл при рН мочи 5 до 200 мг/ дл при рН 7 [11]. Необходимо отметить, что для оценки эффективности и своевременной коррекции проводимой консервативной терапии крайне важно регулярное определение $\mathrm{pH}$ мочи с использованием индикаторных полосок или рН-электродов. Может проводиться как оценка мочи, полученной при однократном заборе материала, так и суточной мочи, что нивелирует влияние циркадных ритмов на результаты исследования $[2,8,9]$.
Основными препаратами, используемыми для консервативного лечения уратов, являются тамсулозин (в том числе в сочетании с калия натрия гидроцитратом), калия цитрат, а также калия цитрат с натрия цитратом.

Было выдвинуто предположение, что применение препаратов калия циттрата для растворения уратов может снижать риск возникновения кальциевых камней [9]. Одним из механизмов действия калия цитрата является повышение рН мочи до значений, необходимых для растворения конкрементов [5]. Данные лекарственные средства являются препаратами выбора для консервативной терапии уратов, так как обладают минимальными побочными эффектами $[13,18]$.

Препараты тамсулозина (Омник $\left.{ }^{\circledast}\right)$, в том числе в комбинации с калия натрия гидроцитратом (Уралит-У®) могут увеличивать частоту спонтанного пассажа конкрементов при локализации уратов в дистальном отделе мочеточника [2]. Данные препараты являются наиболее эффективными при размерах конкрементов в диапазоне 8-11 мм, при отсутствии патологии контралатерального 
мочеточника, наличии гидронефроза легкой или средней степени, а также терапии длительностью до 4 недель или до полного растворения конкрементов [5].

На данный момент недостаточно изучен вопрос эффективности применения аллопуринола у пациентов с уратами, так как в большинство исследований были включены лишь больные с кальциевыми конкрементами. Существует мнение, что назначение данного препарата может быть целесообразно при концентрации МК в моче выше 4 ммоль/л [4] ввиду его гипоурикурического действия [15]. Однако Williams D. I. и соавт. утверждают, что применение аллопуринола у детей снижает продукцию мочевой кислоты, что приводит к алкализации мочи и повышение растворимости конкрементов [23, с. 293].

Метафилактика образования уратов заключается в увеличении диуреза путем потребления большего количества жидкости для образования более 2,5 литров мочи в сутки, предотвращении гиперурикозурии и повышение $\mathrm{pH}$ мочи $[4,9,13,15]$. Рекомендуется снизить потребление животного белка (за исключением молочных продуктов) [13, 14], а также продуктов, содержащих более 150 мг пуринов, и повысить содержание овощей и фруктов в рационе [10].

\section{Эак^ючение}

Учитывая растущую заболеваемость мочекаменной болезнью и распространенность широкого спектра предрасполагающих к ней факторов в популяции, в том числе в детском возрасте, с каждым годом данная тема становится все более актуальной не только для стран с жарким климатом. В свою очередь увеличение доли конкрементов, состоящих из уратов, обуславливает необходимость дальнейших исследований для улучшения методов визуализации и определения наиболее эффективного лечения. В частности, открытым остается вопрос абсолютных и относительных показаний к применению лучевых методов исследования у детей, а также рекомендаций к выбору препаратов для проведения хемолиза.

\section{ЛИТЕРАТУРА}

1. Asi T. et al. Shockwave Lithotripsy for Kidney Stones as a First Line Therapy in Children Younger Than 2 Years //Journal of Pediatric Urology. - 2020.

2. Boari B. et al. Circadian variation in urine pH and uric acid nephrolithiasis risk //Nephrology Dialysis Transplantation. — 2008. — T. 23.— № . 1.—C. 411-412.

3. Cicerello E. Uric acid nephrolithiasis: An update //Urologia Journal. — 2018. — T. 85.— № . 3.— C. 93-98.

4. Courbebaisse M., Prot-Bertoye C., Daudon M. Nephrolithiasis: From mechanisms to preventive medical treatment //Nephrologie \& Therapeutique.- 2020.T. 16. - №. 1.-C. $65-75$.

5. Elbenday M. et al. 1317 Role of combined use of potassium citrate and tamsulosin in the management of uric acid distal ureteral calculi //The Journal of Urology. 2010.

6. Gridley, C.M., Sourial, M.W., Lehman, A., \& Knudsen, B.E. (2019). Medical dissolution therapy for the treatment of uric acid nephrolithiasis. World journal of urology, $1-7$.

7. Hesse A. Urinary stones: Diagnosis, treatment, and prevention of recurrence. - Karger Medical and Scientific Publishers, 2009.

8. Hoppe B., Kemper M. J. Diagnostic examination of the child with urolithiasis or nephrocalcinosis //Pediatric nephrology. — 2010.— - T. 25. — № .3.—C. 403-413.

9. Kamphuis G.M. et al. Method of alkalization and monitoring of urinary $\mathrm{pH}$ for prevention of recurrent uric acid urolithiasis: a systematic review //Translational andrology and urology.—2019.— - T. 8. — № . Suppl 4.—C. S448.

10. Kanbara A., Hakoda M., Seyama I. Urine alkalization facilitates uric acid excretion //Nutrition journal. — 2010. — T. 9. — № . 1.—C. 45.

11. Kenny J.E.S., Goldfarb D. S. Update on the pathophysiology and management of uric acid renal stones //Current rheumatology reports. — 2010.— T. 12.— № . 2.- C. 125-129.

12. Moses R. et al. Changes in stone composition over two decades: evaluation of over 10,000 stone analyses //Urolithiasis. — 2015.— T. 43.— № . 2.—C. 135-139.

13. Ngo T.C., Assimos D. G. Uric acid nephrolithiasis: recent progress and future directions //Reviews in urology.— 2007.— T. 9.— № . 1.—C. 17.

14. Pearle M.S. et al. Medical management of kidney stones: AUA guideline //The Journal of urology.—2014. — T. 192. — № . 2. — C. 316-324.

15. Qaseem A. et al. Dietary and pharmacologic management to prevent recurrent nephrolithiasis in adults: a clinical practice guideline from the American College of Physicians //Annals of internal medicine.— 2014.— T. 161.— № . 9. - C. 659-667.

16. Salem S.M., Sultan M. F., Badawy A. Oral dissolution therapy for renal radiolucent stones, outcome, and factors affecting response: A prospective study //Urology annals. - 2019.—T. 11.— № . 4.—C. 369.

17. Singh S.K., Agarwal M. M., Sharma S. Medical therapy for calculus disease //BJU international.— 2011.— —. 107.— № . 3.—C. 356-368.

18. Skolarikos A. et al. Metabolic evaluation and recurrence prevention for urinary stone patients: EAU guidelines //European urology. — 2015. — T. 67.— № . 4. C. $750-763$.

19. Sorokin I. et al. Epidemiology of stone disease across the world //World journal of urology.— 2017.— T. 35. — № . 9. — C. 1301-1320.

20. Tasian G.E., Copelovitch L. Evaluation and medical management of kidney stones in children //The Journal of urology.— 2014.— —. 192.— № .5.—C. 1329-1336.

21. Thomas C. et al. Urinary calculi composed of uric acid, cystine, and mineral salts: differentiation with dual-energy CT at a radiation dose comparable to that of intravenous pyelography //Radiology.—2010.— T. 257.— № . 2.—C. 402-409. 
22. Trinchieri A., Montanari E. Prevalence of renal uric acid stones in the adult //Urolithiasis. — 2017. — T. 45. — № . 6. — C. 553-562.

23. Williams D.I. et al. Urology in childhood. - Springer Science \& Business Media, 2012.

24. Yadav P. et al. Technique, complications, and outcomes of pediatric urolithiasis management at a tertiary care hospital: evolving paradigms over the last 15 years // Journal of pediatric urology.— 2019.- - T. 15.— №.6.- C. 665. e1-665. e7.

25. Голованов С.А. и др. Индекс массы тела и химический состав мочевых камней //Экспериментальная и клиническая урология.—2015.—№ . 4.—C. 94-99.

26. Голованов С.А. и др. Тенденции распространенности метаболических типов мочекаменной болезни в Московском регионе. Сравнительный анализ за период с 2010 по 2013 гг //Экспериментальная и клиническая урология.— 2014.— № . 4. — С. 54-57.

27. Лазарева Т.С., Застело Е. С., Халецкая 0. В. Влияние характера питания и наследственности на мочевую экскрецию солей у детей с синдромом мальабсорбции //Медицинский альманах.—2018.—№ . 3 (54).

28. Шалденко 0.А. и др. Результаты дистанционной литотрипсии крупных конкрементов почек у детей //Вестник урологии. — 2019.—№ . 2.

29. Эмануэль В.Л. и др. Современное состояние диагностики мочекаменной болезни у детей //Педиатр. — 2014.— Т. 5. — № . 4.

с Бокова Елизавета Олеговна ( eobokova@gmail.com ), Васюкова Олеся Александровна ( o.vas.93@gmail.com ).

Журнал «Современная наука: актуальные проблемы теории и практики»

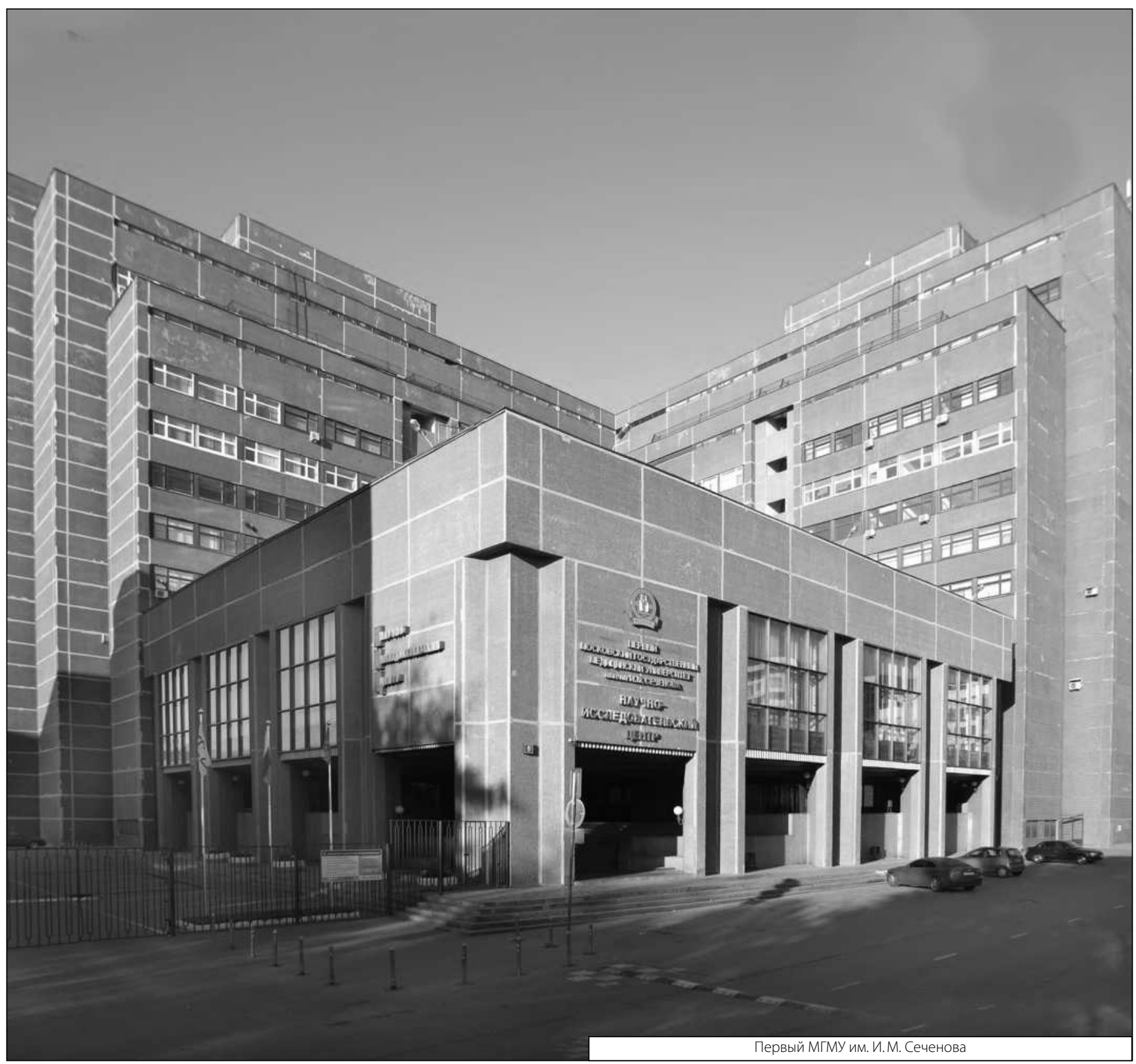

